\title{
Factores de riesgo psicológico-social y desempeño laboral en los doctorandos de educación de una universidad
}

\author{
Format and content criteria in peer review of academic texts with university students
}

\section{Critérios de formato e conteúdo na revisão por pares de textos acadêmicos com estudantes universitários}

ARTÍCULO DE INVESTIGACIÓN

\author{
Dulio Oseda Gago \\ dosedag@unmsm.edu.pe \\ ORCID: 0000-0002-3136-6094
}

Universidad Nacional Mayor de San Marcos, Perú

Marco Antonio Oseda Gago

marco.oseda@unh.edu.pe

ORCID: 0000-0002-7580-8974

Universidad Nacional de Huancavelica
Sonia Gladys Gutiérrez Monzón

c16888@utp.edu.pe

ORCID: 0000-0001-6474-762X

Universidad Tecnológica del Perú. Perú

Nancy Aida Carruitero Ávila

nancyaida29@gmail.com

ORCID: 0000-0002-5138-6519

Universidad César Vallejo

Recibido 11 de enero 2021 | Arbitrado y aceptado 19 de febrero 2021| Publicado en marzo 2021

\section{RESUMEN}

La investigación parte del objetivo: Determinar la relación que existe entre los factores de riesgo psicológico - social y la valoración del desempeño laboral en los doctorandos de Educación de la Universidad César Vallejo de Trujillo en el periodo 2020. La investigación fue del tipo básica y nivel correlacional, de diseño no experimental - transversal, se utilizó el método científico y el método hipotético deductivo, como instrumentos se utilizó el cuestionario de encuesta para ambas variables y se trabajó con un muestreo probabilístico conformado por 232 doctorandos de Educación. La investigación se sustenta en la teoría del desarrollo social de los factores psicológicos y sociales de Jones (2002) y la teoría de la evaluación del talento humano sobre el rendimiento laboral. El aporte más importante de la presente investigación considera que en base a los datos analizados y procesados, con un nivel de significancia del $5 \%$ que existe una relación directa fuerte $(r h o=0,916)$ y altamente significativo entre los factores de riesgo psicológico - social y la valoración del desempeño laboral en los doctorandos de Educación de la Universidad César Vallejo de Trujillo en el periodo 2020 .

Palabras clave: Factor de riesgo; factor psicológico; factor social; valoración del desempeño laboral

\section{ABSTRACT}

The research starts from the objective: To determine the relationship that exists between the psychological-social risk factors and the evaluation of the work performance in the doctoral students of Education of the César Vallejo de Trujillo University in the period 2020. The research was of the basic type and level Correlational, non-experimental - cross-sectional design, the scientific method and the hypothetical deductive method were used, as instruments the survey questionnaire was used for both variables and a probabilistic sampling was made up of 232 doctoral students in Education. The research is based on the theory of social development of psychological and social factors of Jones (2002) and the theory of the evaluation of human talent on work performance. The most important contribution of this research considers that based on the data analyzed and processed, with a significance level of $5 \%$, there is a strong direct relationship (rho $=0.916$ ) and highly significant between the psychological-social risk factors and the assessment of work performance in the doctoral students in Education at the César Vallejo de Trujillo University in the 2020 period.

Key words: Risk factor; psychological factor; social 


\section{RESUMO}

A pesquisa parte do objetivo: Determinar a relação que existe entre os fatores de risco psicossocial e a avaliação do desempenho laboral dos alunos de doutorado em Educação da Universidade César Vallejo de Trujillo no período de 2020. A pesquisa foi de base tipo e nível Correlacional, não experimental - delineamento transversal, utilizou-se o método científico e o método hipotético dedutivo, como instrumentos utilizou-se o questionário survey para ambas as variáveis e uma amostra probabilística foi composta por 232 doutorandos em Educação. A pesquisa é baseada na teoria do desenvolvimento social dos fatores psicológicos e sociais de Jones (2002) e na teoria da avaliação do talento humano no desempenho no trabalho. A contribuição mais importante desta pesquisa considera que com base nos dados analisados e processados, com um nível de significância de $5 \%$, existe uma relação direta forte (rho = $0,916)$ e altamente significativa entre os fatores de risco psicossocial e a avaliação de desempenho no trabalho dos alunos de doutorado em Educação da Universidade César Vallejo de Trujillo no período de 2020.

Palavras-chave: Fator de risco; fator psicológico; fator social; avaliação de desempenho de trabalho

\section{INTRODUCCIÓN}

Los factores psicosociales como elementos externos afectan de manera directa a los trabajadores en sus organizaciones los cuales afectan a la relación persona con su grupo social cuya presencia puede llegar a causar un desequilibrio psicológico en el individuo y de la misma manera un ambiente tenso estresante a nivel organizacional (Ramírez, 2018).

En esa óptica, la disciplina que aborda directamente el estudio de los riesgos psicosociales en el centro laboral, en su entorno laboral y de la calidad de vida laboral es la Psicología de la Salud Ocupacional (PSO), que tiene como principal objetivo que las personas puedan trabajar dentro de un ambiente positivo saludable, de tal manera que puedan producir en la organización, atender a los demás, que puedan desarrollarse tanto profesional como laboralmente, y que su trabajo sea recompensado valorado y además de tener la posibilidad de utilizar su talento, sus conocimientos, $\mathrm{y}$ sus destrezas $\mathrm{y}$ habilidades, como vía para alcanzar un alto rendimiento en su organización, al tiempo que perciben alta satisfacción laboral y bienestar en el trabajo (Mansilla y Favieres, 2012).

Los factores psicosociales se pueden evidenciar en la carga de trabajo, largas jornadas que son exhaustivas de la misma manera sus hábitos de trabajo estos que no son adecuados, el reconocimiento del trabajo que en la mayoría de las veces no es bien recompensado por lo tanto no poseen incentivo emocional el apoyo social igual viene a ser intenso y esto afecta en los trabajadores provocando estrés ya que no poseen apoyo de los supervisores sino más bien exceso de supervisión y otros causante más.

Cuando estos factores exceden la capacidad del trabajador pueden ocasionar un grave daño y hacerlos vulnerables a los trabajadores. Las nuevas tecnologías son sin duda eficaces ya que permiten el potencial y aumento de la productividad, pero en cierta forma ha llevado a que se incremente una tasa de prevalencia de algunos riesgos psicosociales los cuales afectan a las condiciones presentes en una situación laboral y se relacionan directamente con las condiciones de la tarea como es estilo de mando, carga de trabajo, autonomía, el contenido de la tarea e incluso el entorno, que tiene la capacidad de afectar al desarrollo del trabajo, y más todavía en estos tiempos de pandemia que todo el trabajo se ha virtualizado de manera forzosa.

La promoción de la salud en el lugar de trabajo es fundamental para la prevención de trastornos psicológicos y sociales, así como también para promover una mejor salud mental. Así también se ha provocado cambios en las jornadas de trabajo, exposición a altos niveles de estrés y cambios extenuantes para los trabajadores, lo que ha generado el 
aumento de factores de riesgos psicosociales y sociales, que pueden afectar su desempeño laboral en sus centros de trabajo.

De esta manera el medio ambiente laboral puede influir en el bienestar de los trabajadores, es decir, cuando las condiciones de trabajo son buenas, los efectos para la salud son positivos, los trabajadores se sienten satisfechos y son menos propensos a sufrir algún accidente, y por ende en su desempeño en su centro laboral; y precisamente este fenómeno se ha visto reflejado en los doctorandos de Educación de la Universidad César Vallejo, quienes a parte de estudiar se desempeñan laboralmente en las instituciones educativas de la Educación Básica Regular y también en la educación superior universitaria y no universitaria tanto pública como privada; todo esto se percibe cuando las condiciones de trabajo son desfavorables los trabajadores se sienten menos involucrados con sus actividades, lo que provoca falta de interés por el desempeño de sus tareas y los hace propensos a padecer de estrés laboral crónico.

La presente investigación tiene por finalidad investigar los factores de riesgo psicológico y social que repercuten muchas veces en el desempeño laboral como variables, que busca la relación de ambos en la población escogida y la importancia de la viabilidad del método de evaluación que se realizaron en el presente año, permitiendo respectivamente recopilar antecedentes en primer lugar antecedentes sobre los mismos.

Según el marco legal, de la Ley 29783 de Seguridad y Salud en el Trabajo, esta establece en su connotación dogmática y doctrinaria la obligación del empleador de identificar los riesgos a los que está expuesto el trabajador en su diario quehacer, asimismo a eliminar los evitables que pudieran suscitarse, y así mismo a evaluar aquellos riesgos que no se puedan evitar a fin de obtener la información necesaria para poder planificar las actividades preventivas en un sistema de seguridad.

Por lo dicho, las organizaciones públicas y privadas de todos los sectores deben prestar especial atención a los diversos factores de riesgo psicológico y social que se presentan en el trabajo, estos riesgos son importantes frente a los diversos fenómenos de carácter físico, químico, biológico y disergonómicos; por lo tanto, el ser humano según la gestión del talento humano es la pieza fundamental para prevé riesgos laborales, en nuestro caso en los doctorandos de Educación de la Universidad César Vallejo de Trujillo.

Al respecto, Navarro, experto en Psicometría de la Seguridad Ocupacional de la Universidad Politécnica de Madrid (2010) considera, que al menos el $80 \%$ de los accidentes laborales tiene como causa principal el comportamiento humano, el cual es corroborado por múltiples tratados $\mathrm{y}$ experiencias directas asumidos como estudios pilotos en el ambiente organizacional, principalmente en el sector privado.

Tipán (2020) en su investigación: Factores psicosociales y rendimiento laboral de la empresa EFIKAS. El análisis inferencial mostró una fuerte relación, estadísticamente significativa, entre todos los factores psicosocial y el rendimiento laboral, con valores de Chi cuadrado ubicados alrededor de 0,000 . Las variables de condiciones del lugar de trabajo, la carga de trabajo, el contenido y las características de las tareas, las exigencias del trabajo, el papel laboral y desarrollo de la carrera, así como la remuneración y el rendimiento influyen en el rendimiento laboral de los colaboradores de le empresa mencionada. 
Asimismo, Varela (2020) en su investigación: Identificación de los factores de riesgo psicosocial para mejorar el desempeño laboral de los trabajadores durante el periodo 2020-2021. Los riesgos psicosociales se pueden presentar en mayor o menor grado, todo esto podrá depender de la naturaleza de la empresa por tal razón es necesario elaborar el análisis del riesgo para de esta manera evitar afecciones a la salud de los trabajadores, así como evitar la rotación del personal.

Al respecto Ramírez (2018) realizó la investigación factores de riesgos psicosociales en el personal de enfermería del servicio de Emergencia de un Hospital Público de la ciudad de Quito. La investigación es de tipo exploratoria descriptiva no correlacional, el método de estudio es inductivo, deductivo y estadístico; la población de estudio 35 enfermeras que corresponde al 100\% del personal investigado. Se aplicó el Cuestionario (FPsico 3.1) factores de riesgo psicosociales. Se concluye que existe la evidencia de riesgo psicosocial asociado con las demandas psicológicas dentro del personal de servicio asignado.

Y luego Molina (2018) en su investigación: Los factores de riesgo psicosocial y la salud ocupacional de los servidores públicos de la Facultad de Ciencia e Ingeniería en Alimentos de la Universidad Técnica de Ambato. la metodología utilizada se desarrolla en el enfoque cuali-cuantitativo, permitiendo recopilar datos relevantes a través de una encuesta validada con un estudio estadístico de fiabilidad "Alfa de Cronbach", así mismo, se dispone de valores numéricos para su respectiva tabulación, desarrollando el método estadístico "Correlación de Variables de Pearson" y "Coeficiente de Correlación "R" de Pearson" para su verificación. De esta manera se constituirá conclusiones en el cual se pretende dar una explicación a las variaciones del entorno laboral que un trabajador experimenta y debe manejar para ofrecer el mejor rendimiento a su institución.

Respecto a las bases teórico-científicas, los factores de riesgo laboral, tanto psicológico y social son condiciones que existen en el trabajo diario que de no ser eliminados tendrán como consecuencia accidentes laborales y enfermedades profesionales precisa el Ministerio de Trabajo y Promoción del Empleo (2014). Se relacionan siempre con una probabilidad y unas consecuencias. Los factores de riesgo deben ser minimizados o eliminados con prevención y protección. Del estudio de factores de riesgo se encargan la higiene, la medicina del trabajo, la ergonomía y la psicología que actúan como un conjunto multidisciplinario para así poder llegar al objetivo de mantener la salud para los trabajadores, además de leyes que permitan el cumplimiento de los procedimientos de seguridad tanto para el trabajador como para las organizaciones.

Según Vivaldi y Barra, (2012) los factores psicosociales son condiciones presentes en situaciones laborales relacionadas con la organización del trabajo, el tipo de puesto, la realización de la tarea, e incluso con el entorno; que afectan al desarrollo del trabajo y a la salud de las personas trabajadoras, y luego Rodríguez, Sánchez, Dorado, y Ramírez, (2015) sostienen que los riesgos psicosociales son un fenómeno multifactorial que afectan tanto la salud física y mental de los trabajadores como su desempeño.

La Teoría de los 2 factores de Herzberg, en esta teoría se explica el comportamiento de las personas en el trabajo y plantea la existencia de dos factores que orientan el comportamiento de las personas, el principal factor tiene el logro de los objetivos como 
factores extrínsecos que viene a ser el ambiente y las condiciones físicas de su trabajo, Carayon, Haims, y Yang, (2017); Melía, (2016). En cambio, los factores intrínsecos o motivacionales son aquellos que están controlados por las personas ya que estas son actividades desempeñadas por el trabajador el cual influye en la satisfacción de logro de cada actividad realizada; de la misma manera menciona a los factores de insatisfacción que son aquellos que provocan inconformidades del trabajador en cuanto a los factores extrínsecos como son el salario las prestaciones el horario de trabajo etc.

Con estas bases nos damos cuenta de la importancia que tiene estos factores dentro de las organizaciones y como esto influye en la mejora de sus trabajadores y el logro de sus objetivos de manera satisfactoria. Por ello tenemos como principales factores a los aspectos higiénicos; salario, beneficios sociales, tipo de dirección o supervisión que las personas reciben de sus superiores y de cómo deben trabajar dentro de las organizaciones, las condiciones físicas y ambientales de trabajo estas deben ser las adecuadas para un mejor clima laboral, las políticas físicas de las organizaciones, reglamentos internos que deben ser cumplidos por el personal de servicio entre otros (Politécnico Grancolombiano, 2017).

Por otro lado, para Quispe y Vigo, (2017) la labor del docente es significativa en este proceso, pues él es, el que propicia el conocimiento en el estudiante y el que motiva estratégicamente a alcanzar las metas educativas propuestas por cada centro de estudios. Es aquí donde el desempeño óptimo del docente se evidencia en su capacidad de integrar no solo a los estudiantes para que sea un aprendizaje organizativo si no también, integración de los nuevos conocimientos para ser aplicados en la cotidianidad. Un docente con alto desempeño laboral es un profesional competente y comprometido con la educación, que otorga oportunidades de aprendizaje a todos los estudiantes (teniendo en cuenta las necesidades) y a través de su formación profesional, la cual se fundamenta en su propia formación personal, contribuye a construir la sociedad que queremos como país.

Para Soto, (2017) una organización de aprendizaje es capaz de crear, adquirir, transmitir y reformar actitudes. Cuando se aprende individualmente, el capital intelectual de la organización cada vez es mayor, correspondiéndole a la gestión convertirla en acción. A mayor capacidad de aprendizaje de la organización, mayor es el desarrollo organizacional.

El desempeño laboral docente radica en su formación, que es un desarrollo progresivo y de organización de la persona, que lo conduce a consolidar aprendizajes para dar a conocer a otros. Marcelo (1995) distingue otros tipos de formación: Autoformación, heteroformación e interformación. La autoformación es una formación en la que la persona participa de forma independiente y teniendo bajo su propio control los objetivos, los procesos, los instrumentos, y los resultados de la propia formación. La heteroformación es una formación que se organiza y desarrolla por especialistas sin que se vea comprometida la personalidad del sujeto que participa. La interformación se define como la acción educativa que se ejerce entre los futuros enseñantes o entre maestros.

La riqueza de ser seres sociales por naturaleza hace que nuestra interacción con nuestro entorno promueva contextos de aprendizaje que favorecen la formación compleja de cada persona. El papel fundamental de la evaluación, en la actualidad, 
es expandir las oportunidades para que los docentes reflexionen sobre la enseñanza y examinar analíticamente el proceso docente educativo. Por esa razón, es muy importante el uso de la autoevaluación en todo sistema de evaluación del desempeño profesional del docente que se desee implementar (Valdez, 2009).

Como actualmente no existe una articulación entre las organizaciones públicas $\mathrm{y}$ privadas al respecto, se hace necesario diagnosticar y a partir de ellos tomar decisiones en situaciones similares; por lo tanto, esta investigación aborda la problemática de la limitada atención en la prevención de los factores de riesgos psicológico, social y la valoración del desempeño laboral.

- Problema: ¿De qué manera los factores de riesgo psicológico y social se relacionan con la valoración del desempeño laboral en los doctorandos de Educación de la Universidad César Vallejo de Trujillo?
- Objetivo: Determinar la relación que existe entre los factores de riesgo psicológico y social con la valoración del desempeño laboral en los doctorandos de Educación de la Universidad César Vallejo de Trujillo.

- Hipótesis: Existe una relación directa y significativa entre los factores de riesgo psicológico y social, con la valoración del desempeño laboral en los doctorandos de Educación de la Universidad César Vallejo de Trujillo.

\section{METODOLOGÍA}

El tipo de investigación fue básica, pues se utilizaron variables ya conocidas que tienen el sustento de teorías, asimismo el nivel de investigación fue el correlacional. En la investigación, la población estuvo constituida por 378 doctorandos de Educación de la Universidad César Vallejo de Trujillo en el periodo 2020. La muestra fue probabilística, y estuvo conformada por 191 doctorandos. Se utilizó el diseño descriptivo - correlacional, cuyo esquema es el siguiente:

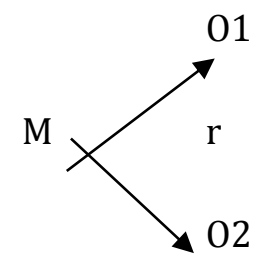

Dónde: M: Muestra

$\mathrm{O}_{1}$ : Medición de la variable 1.

$\mathrm{O}_{2}$ : Medición de la variable 2 .

r: Relación de las dos variables de estudio. 
La técnica empleada para la recolección de datos fue la encuesta, y para la confiabilidad de los instrumentos se utilizó el alfa de Cronbach donde se obtuvo el valor de 0,978 en el instrumento factores de riesgo psicológico-social y en la otra variable fue de 0,956 en la variable desempeño laboral, cuyos resultados dieron como confiables a ambos instrumentos para su respectiva aplicación.

Para la validación de los instrumentos de investigación se utilizó el método de Análisis de Componentes Principales, donde se forma una combinación lineal de las variables observadas. El primer componente principal fue la combinación que da cuenta de la mayor cantidad de la varianza en la muestra. El segundo componente principal responde a la cantidad de varianza inmediatamente inferior a la primera y no está correlacionado con el primero. Los valores superiores al $20 \%$ en el primer componente expresan unicidad de componentes en la dimensión, de esta mayor a mayor valor mayor grado de unicidad. El instrumento de investigación para medir la inteligencia emocional y procrastinación poseen una excelente validez de constructo debido a que los ítems que lo componen están estrechamente vinculados (Feuerstein, Rand, Hoffman, y Miller, 1980).

Tabla 1. KMO y Prueba de Bartlett

Medida de adecuación muestral de Kaiser-Meyer-Olkin.

Prueba de esfericidad de Bartlett

La medida de adecuación muestral del test de Kaiser - Meyer - Olkin es de 0.626, al ser mayor a 0.5 se afirma que el valor es regular, en consecuencia, se puede continuar el análisis de los ítems de esta variable, es decir que la muestra se adecúa ajustadamente al tamaño del instrumento. La prueba de esfericidad de Bartlett mide la asociación entre los ítems de cada una de las dimensiones, se determina si los ítems están asociados entre sí, con una significancia que debe ser inferior a 0.05. En el caso planteado la significancia es de 0,000 rechazándose la hipótesis nula, por lo que se concluye que la correlación de la matriz no es una correlación de identidad. Es decir, que los ítems están asociados hacia la medición de una sola identidad.
El método de Comunalidad permitió extraer la proporción de varianza explicada por los factores de cada ítem, valores pequeños indican que el ítem estudiado no debería ser tomado en cuenta para el análisis final. La comunalidad expresa la parte de cada variable (su variabilidad) que puede ser explicada por los factores comunes a todas ellas, es decir, aquellos que consideramos como parte de la dimensión de estudio (Feuerstein, Rand, Hoffman, y Miller, 1980).

Además, para la aplicación de los instrumentos se tuvo el criterio de consentimiento informado (Kerlinger y Lee, 2002), además para la contrastación de la hipótesis se empleó la estadística inferencial de la prueba rho de Spearman. 


\section{RESULTADOS}

Antes de describir a las variables de estudio, debemos precisar los datos de la muestra de estudio:

Tabla 2. Datos de la edad cronológica

\begin{tabular}{|c|c|c|}
\hline Intervalos de edad & Frecuencia & Porcentaje \\
\hline De 18 a 28 & 1 & 0.52 \\
\hline De 29 a 38 & 56 & 29.32 \\
\hline De 39 a 48 & 112 & 58.64 \\
\hline De 49 a 59 & 19 & 9.95 \\
\hline Mayor de 60 & 3 & 1.57 \\
\hline Total & 191 & 100.00 \\
\hline
\end{tabular}

De la tabla 2, se puede apreciar que, respecto a la edad cronológica de los doctorandos de la muestra de estudio, se tiene que la mayoría de ellos tienen edades que fluctúan entre 39 y 48 años, siendo este el 58,64\%, seguido de las edades entre 29 y 38 con el 29,32\% y finalmente se tiene solo 3 casos cuyas edades son mayores de 60 años. Como se puede apreciar en los doctorandos de Educación de la muestra de estudio, la mayoría de ellos tienen edades que fluctúan entre 39 y 48 años, y este recurso humano ya tiene experiencia laboral y muchos de ellos ya ocupan cargos de dirección.

Tabla 3. Condición laboral

\begin{tabular}{|c|c|c|}
\hline Condición & Frecuencia & Porcentaje \\
\hline Sin empleo & 6 & 3.14 \\
\hline Contratado a tiempo definido & 123 & 64.40 \\
\hline Contratado a tiempo indefinido & 7 & 3.66 \\
\hline Nombrado & 54 & 28.27 \\
\hline Cesante / jubilado & 1 & 0.52 \\
\hline Total & 191 & 100.00 \\
\hline
\end{tabular}

De la Tabla 3, se puede apreciar que, respecto a su condición laboral de los doctorandos de la muestra de estudio, 123 doctorandos que representa el $64.40 \%$ están en condición de contratados a tiempo definido, 54 doctorandos que representa el 28,27 en su condición de nombrados, y lo que preocupa si es que se tiene a 6 doctorandos que representa el 3,14 a la fecha sin empleo. A manera de síntesis, la mayoría de los participantes de la muestra de estudio están en condición de docentes contratados en sus instituciones educativas. 
Tabla 4. Datos del sexo de los trabajadores

\begin{tabular}{lccc}
\hline & Intervalos de edad & Frecuencia & Porcentaje \\
\hline Femenino & & 136 & 71.20 \\
Masculino & 55 & 28.80 \\
& \multirow{2}{*}{ Total } & 191 & 100.00 \\
\hline
\end{tabular}

De la Tabla 4, se puede apreciar que, respecto al sexo de los doctorandos de la muestra de estudio, se tiene que la mayoría de ellos son del sexo femenino con 136 casos siendo este el $71,20 \%$, y el sexo femenino con 55 casos, siendo este el $28,80 \%$. Como se puede apreciar, el sexo femenino es el predominante en los estudios del doctorado en la universidad. Ahora si veamos el detalle de las dos variables de estudio.

Los resultados de la primera variable de estudio, es decir el cuestionario sobre los factores de riesgo psicológico y social aplicado a la muestra de estudio.

Tabla 5. Niveles de la Variable 1: Cuestionario de factores de riesgo psicológico y social

\begin{tabular}{lccc}
\hline & Niveles & Frecuencia & Porcentaje \\
\hline Ninguno & & 0 & 0.00 \\
Leves & 30 & 15.71 \\
Moderados & & 153 & 80.10 \\
Crónicos & & 8 & 4.19 \\
& Total & $\mathbf{1 9 1}$ & $\mathbf{1 0 0 . 0 0}$ \\
& & & \\
\hline
\end{tabular}

De la tabla 5 , se puede apreciar que en la variable 1 denominada factores de riesgos psicológico y social, de los 191 doctorandos encuestados, 153 de ellos que representa el $80,10 \%$ consideran que dichos riegos son moderados, asimismo 30 de ellos que es el $15,71 \%$ menciona que dichos factores de riesgo son leves y 8 doctorandos que es el $4,19 \%$ sostienen que el nivel de factores de riesgo es crónico el cual si es preocupante. A manera de síntesis, se puede apreciar que el nivel predominante es el moderado, es decir, existe un alto índice de doctorandos cuyo factor de vulnerabilidad psicológico y social está siendo vulnerado (Camacho, 2013; Coral, 2014) en este contexto actual sería por el trabajo remoto que realizan en estos tiempos de pandemia.

Los resultados de la segunda variable de estudio, es decir los Cuestionario de la valoración del desempeño laboral aplicado a la misma muestra de estudio. 
Tabla 6. Niveles de la Variable 2: Valoración del desempeño laboral

\begin{tabular}{|c|c|c|}
\hline Niveles & Frecuencia & Porcentaje \\
\hline Bajo & 0 & 0.00 \\
\hline Medio & 17 & 8.90 \\
\hline Bueno & 116 & 60.73 \\
\hline Muy bueno & 58 & 30.37 \\
\hline Total & 191 & 100.00 \\
\hline
\end{tabular}

De la Tabla 6, se puede apreciar que en la variable 2 denominada valoración del desempeño laboral, de los 191 doctorandos de la muestra de estudio, 116 de ellos que representa el $60,73 \%$ consideran que dicha valoración es buena, asimismo 58 de ellos que es el 30,37\% menciona que dicha valoración es muy buena y solo 17 trabajadores que es el $8,90 \%$ sostienen que el nivel de valoración del desempeño laboral es medio o regular. A manera de síntesis, se puede apreciar que el nivel predominante de la valoración del desempeño laboral es el bueno, el cual es satisfactorio, a pesar de las dificultades y limitaciones que trae consigo el trabajo en entornos remotos en tiempos de pandemia.

Ahora bien, el proceso que permite realizar el contraste de hipótesis requiere ciertos procedimientos. Se ha podido verificar los planteamientos de diversos autores y cada uno de ellos con sus respectivas características y peculiaridades, motivo por el cual era necesario decidir por uno de ellos para ser aplicado en la investigación.

Tabla 7. Coeficiente de correlación de la hipótesis de investigación

\begin{tabular}{ccccc}
\hline & & & $\begin{array}{c}\text { Factores de riesgo } \\
\text { psicológico y social }\end{array}$ & $\begin{array}{c}\text { Valoración del } \\
\text { desempeño } \\
\text { laboral }\end{array}$ \\
\hline $\begin{array}{c}\text { Spearman's } \\
\text { rho }\end{array}$ & $\begin{array}{c}\text { Factores de riesgo } \\
\text { psicológico y social }\end{array}$ & $\begin{array}{c}\text { Correlation } \\
\text { Coefficient }\end{array}$ & 1.000 & $.928\left(^{* *}\right)$ \\
& Sig. (2-tailed) &. & .000 \\
& Naloración del & $\begin{array}{c}\text { Correlation } \\
\text { Coefficient }\end{array}$ & $.928\left(^{* *}\right)$ & 191 \\
& & Sig. (2-tailed) & .000 & 1.000 \\
& & $\mathrm{~N}$ & 191 &. \\
\hline
\end{tabular}

** Correlation is significant at the 0.01 level (2-tailed). 
Ahora bien, teniendo como referencia a (Hernández, Fernández y Baptista, 2014) se tiene la siguiente equivalencia (p. 453):

Correlación negativa perfecta: -1

Correlación negativa muy fuerte: $-0,90$ a -0,99

Correlación negativa fuerte: $-0,75$ a -0,89

Correlación negativa media: $-0,50$ a -0,74

Correlación negativa débil: $-0,25$ a $-0,49$

Correlación negativa muy débil: $-0,10$ a $-0,24$

No existe correlación alguna: $-0,09$ a $+0,09$

Correlación positiva muy débil: $+0,10$ a $+0,24$

Correlación positiva débil: $+0,25$ a $+0,49$

Correlación positiva media: $+0,50$ a $+0,74$

Correlación positiva fuerte: $+0,75$ a $+0,89$

Correlación positiva muy fuerte: $+0,90$ a $+0,99$

Correlación positiva perfecta: +1

\section{Planteamiento de hipótesis}

- Hipótesis nula: $\mathrm{H}_{0}$ : No existe una relación directa y significativa entre los factores de riesgo psicológico y social, con la valoración del desempeño laboral en los doctorandos de Educación de la Universidad César Vallejo de Trujillo.

- Hipótesis alterna: $\mathrm{H}_{1}$ : Existe una relación directa y significativa entre los factores de riesgo psicológico y social, con la valoración del desempeño laboral en los doctorandos de Educación de la Universidad César Vallejo de Trujillo.

\section{Nivel de significancia o riesgo}

$$
\begin{aligned}
& \alpha=0,05 . \\
& \mathrm{gl}=191-1=190
\end{aligned}
$$

Valor crítico $=1,96$

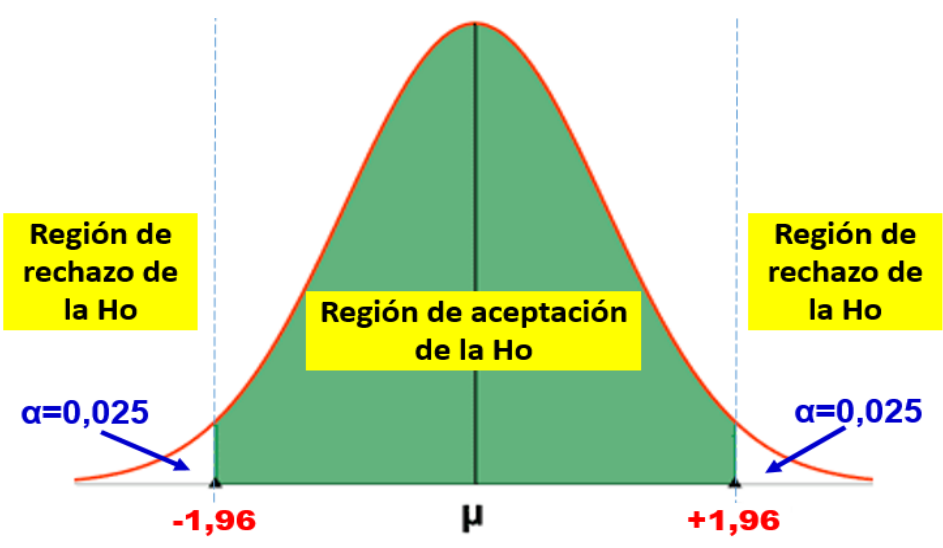


- Decisión estadística: Puesto que p-valor: $0,000<0,010$; en consecuencia, se rechaza la hipótesis nula (Ho) y se acepta la hipótesis alterna (Hi).

- Conclusión estadística: Se concluye que existe relación directa muy fuerte $y$ altamente significativo entre los factores de riesgo psicológico y social, con la valoración del desempeño laboral en los doctorandos de Educación de la Universidad César Vallejo de Trujillo.

\section{DISCUSIÓN}

De las tablas visualizadas se deduce que los factores de riesgo psicológico y social tienen una relación directa (positiva) fuerte, y altamente significativo con la valoración del desempeño laboral; sin embargo, se ha podido observar, primero respecto a la aplicación de nuestros instrumentos de investigación, el temor e indiferencia (Jaén, 2010) que muestran algunos estudiantes a ser encuestados, en dar a conocer sus actitudes reales con respecto a los factores de riesgo y también a su desempeño laboral en sus centros laborales (OIT, 1986).

Es así que, según los resultados de la encuesta virtual aplicado en el Google formulario, se pudo observar que la mayoría de los doctorandos encuestados manifestaron que los factores de riesgo psicológico y social es moderado, alcanzado este el $80,10 \%$. Asimismo, el nivel predominante de la valoración del rendimiento laboral o desempeño labora fue el bueno siendo este el $60.73 \%$, el cual es satisfactorio.

Al respecto, Quispe (2016) en su investigación: Factores de riesgo psicosocial que afectan el desempeño laboral Hospital Nacional Guillermo Almenara Irigoyen 2016, se deduce que los factores de riesgo psicosocial que afectan el desempeño laboral en los trabajadores de la organización, presentan un nivel de exposición más desfavorable para la salud, como las exigencias psicológicas, con un $67,6 \%$ y la inseguridad con un 55,9\%; algo similar a los encontrados en nuestros resultados, principalmente en el componente psicológico como lo ratifica (Blakman, 2014).

Luego Mollo (2015) en su investigación: Relación entre los factores psicosociales y la retroalimentación laboral en asesores de riesgos del área comercial de Lima de una compañía de seguros del Perú; en esta investigación también concluye que existe relación significativa entre los factores psicosociales según la retroalimentación laboral positiva en asesores de riesgos; muy similar a los encontrados en nuestros resultados. Si bien es cierto en nuestras instituciones recién en estos últimos meses del este año 2020 se ha podido evidenciar que las organizaciones públicas ya se vienen estandarizando medidas de seguridad y protocolos en los trabajos remotos $\mathrm{y}$ principalmente en aquellos que cumplen funciones administrativas que son semipresenciales.

Asimismo, nuestros resultados, también coinciden con los obtenidos por Bakovic, (2014) y Cox y Griffiths (2016) donde ambos concluyen que la revisión de los procedimientos establecidos por la organización para asegurar la claridad y comprensión de los mismos por parte de los trabajadores es indispensable, luego el trabajo con los jefes para mejorar sus habilidades en la supervisión del área y equipo de trabajo también se hace necesario, lo mismo pensamos nosotros respecto a los resultados de los riesgos psicológicos y sociales en los doctorandos de Educación de la Universidad César Vallejo.

También, con los hallazgos de Yactayo, (2015) en su tesis: Aplicación de los 
instrumentos para la evaluación del riesgo psicosocial, en la mejora continua a la prevención y control en una organización, en donde concluye que la evaluación de riesgos psicosociales es una necesidad cada vez más sentida y demandada en las organizaciones, dentro de los procedimientos de evaluación de riesgos que exige la Ley SST, como lo sostiene también (Badillo y Jumbo, 2015) muy similar a lo encontrado en los sujetos de nuestra muestra de estudio por la normatividad vigente peruana.

Además, añade, que se debe recordar que el origen del estudio de los factores psicosociales laborales está ligado a la literatura del estrés (Mintzberg, 2017), término introducido en la medicina desde la ingeniería (Motowildo, 2010) con el que también estamos de acuerdo.

\section{CONCLUSIONES}

Se ha determinado con un nivel de significancia del $5 \%$ que existe una correlación directa muy fuerte (rho $=0,916)$ y altamente significativo ( $p$-valor: $0,000<0,010)$ entre los factores de riesgo psicológico y social, con la valoración del desempeño laboral en los doctorandos de Educación de la Universidad César Vallejo de Trujillo, es decir a mayor control de los riesgos laborales psicológicos y sociales, mejor será la valoración del desempeño laboral y viceversa.

Asimismo, se ha determinado que existe una correlación directa muy fuerte (rho $=0,951)$ y altamente significativo entre los factores de riesgo psicológico, con la valoración del desempeño laboral en los doctorandos de Educación de la Universidad César Vallejo de Trujillo, es decir a mayor control de los riesgos laborales psicológicos, mejor será la valoración del desempeño laboral y viceversa.
Finalmente, se ha determinado que existe una correlación directa fuerte (rho $=0,822)$ y altamente significativo entre los factores de riesgo social, con la valoración del desempeño laboral en los doctorandos de Educación de la Universidad César Vallejo de Trujillo, es decir a mayor control de los riesgos laborales sociales, mejor será la valoración del desempeño laboral y viceversa.

\section{REFERENCIAS}

Badillo, G. y Jumbo, L. (2015). Valoración de la influencia de los factores de riesgo psicosocial en el desempeño laboral de agentes de seguridad de entidades financieras del sector la Villaflora en el Sur del distrito Metropolitano de Quito. Quito: Universidad Politécnica Salesiana sede Quito

Bakovic, K. (2014). Factores psicosociales relacionados a accidentes laborales en el contexto de la minería peruana. Lima: Pontificia Universidad Católica del Perú

Blakman, T. (2014). Como afectan los riesgos psicosociales en el rendimiento laboral de los conductores de la empresa de transporte Mamut Andino. Guayaquil: Escuela de Post Grado de la Universidad de Guayaquil

Camacho, J. (2013). Desarrollo de un modelo de gestión para la evaluación de factores de riesgo psicosocial en los conductores de la empresa pública metropolitana de transporte de pasajeros de Quito (Trolebús). Quito: Unidad de Post Grado de la Escuela Politécnica Nacional

Carayon, P., Haims, M. C., y Yang, C. L. (2017). Psychosocial work factors and work organization. In W. Karwowski (Ed.). The International Encyclopedia of Ergonomics and Human Factors (pp. 111-199). London: Taylor y Francis

Coral, M. (2014). Análisis, evaluación y control de riesgos disergonómicos y psicosociales en una empresa de reparación de motores eléctricos. Lima: Pontificia Universidad Católica del Perú 
Cox, T. y Griffiths, A. J. (2016). The assessment of psychosocial hazards at work. In M. J. Schabracq, J. A. M. Winnubst, y C. L. Cooper (Eds.), Handbook of Work and Health Psychology. (pp. 127-146). Chichester: Wiley and Sons

Feuerstein, R., Rand, Y., Hoffman, M. B., y Miller, R. (1980). Instrumental Enrichment: An intervention program for cognitive modifiability. Baltimore: University Park Press

Hernández, R., Fernández, C. y Baptista, P. (2014). Metodología de la Investigación. ( $6^{\circ}$ Ed.) México: Mc Graw Hill

Jaén, M. (2010). Predicción del rendimiento laboral a partir de indicadores de motivación, personalidad y percepción de factores psicosociales. Lima: Escuela de Post Grado Universidad Complutense de Madrid

Kalimo, R., El-Batawi, M., y Cooper, C. L. (1988). Los factores psicosociales en el trabajo y su relación con la salud. Ginebra: OMS

Kerlinger, F. y Lee, H. (2002). Investigación del Comportamiento. México DF: Intermaericana S.A.

Mansilla, F. y Favieres, A. (2012). Factores de riesgo psicosocial en el trabajo. Madrid: Salud.

Marcelo, C. (1995). Formación del profesorado para el cambio educativo. México DF: Universidad Pedagógica Nacional. Recuperado de https://www.researchgate.net/profile/Car los_Marcelo/publication/256194929_For macion_del_Profesorado_para_el_Cambio_E ducativo/links/02e7e521f1a3f866d20000 00/Formacion-del-Profesorado-para-elCambio-Educativo.pdf

Melía, J. (2016). Principios comunes para la evaluación de los riesgos psicosociales en la empresa. Madrid: Foment del Treball Nacional

Ministerio de Trabajo y Promoción del Empleo (2014). Informe Técnico de los Factores de Riesgo Psicosocial en trabajadores de Lima Metropolitana. Consejo Regional de Seguridad y Salud en el Trabajo de Lima Metropolitana. Perú
Mintzberg, H. (2017). El Proceso Estratégico: Conceptos, Contextos y Casos. Bogotá: Prentice Hall Hispanoamericana

Molina, R. A. (2018). Los factores de riesgo psicosocial y la salud ocupacional de los servidores públicos de la Facultad de Ciencia e Ingeniería en Alimentos de la Universidad Técnica de Ambato. Ambato: Universidad Técnica de Ambato

Mollo, M. (2015). Relación entre los factores psicosociales y la retroalimentación laboral en asesores de riesgos del área comercial de Lima de una compañía de seguros del Perú. Lima: Escuela de Post Grado de la Universidad Nacional mayor de San Marcos Motowildo, S. (2010). Handbook of psychology: industrial and organizational psychology

OIT (1986). Factores psicosociales en el trabajo: reconocimiento y control. Ginebra: OIT

Politécnico Grancolombiano (2017). Factores motivacionales e higiénicos de Herzberg en las empresas. Bogotá: Mc Graw Hill. Obtenido de http://sigma.poligran.edu.co/politecnico/a poyo/administracion/admon1/pags/juego $\% 20$ carr era\%20de\%20observacion/HERZBERG2.h tml

Quispe, C. M. (2016) Factores de riesgo psicosocial que afectan el desempeño laboral Hospital Nacional Guillermo Almenara Irigoyen 2016. Pontificia Universidad católica del Perú

Quispe, S. y Vigo, E. (2017). La interacción del aprendizaje organizacional y el desempeño laboral de los educadores de las instituciones educativas públicas. Revista de Investigación en Psicología. 20(2), 407 422

Ramírez, G. (2018). Factores de Riesgos Psicosociales en el personal de enfermería del servicio de Emergencia de un Hospital Público de la Ciudad de Quito. Quito: Universidad Central del Ecuador.

Rodríguez, E., Sánchez-Gómez, J., Dorado, H. A., y Ramírez, J. M. (2015). Factores de riesgo psicosocial intralaboral y grado de estrés en docentes universitarios. Revista 
Colombiana de Salud Ocupacional, 4(2), 1217

Soto, R. (2017). El aprendizaje organizacional para el cambio educativo en una escuela pública del nivel medio superior. México DF: Universidad Pedagógica Nacional. Recuperado de https://repositorio.itesm.mx/ortec/

Tipán, E. F. (2020). Factores psicosociales y rendimiento laboral de la empresa EFIKAS. Quito: Universidad Central del Ecuador

Universidad Politécnica de Madrid. (2010) El factor humano, pieza clave en la prevención de riesgos laborales. Escuela Técnica Superior de Ingenieros Industriales. España

Valdez, H. (2009). Manual de buenas prácticas de evaluación del desempeño profesional de los docentes. Lima: Tarea Asociación Gráfica Educativa

Varela, C. (2020). Identificación de los factores de riesgo psicosocial para mejorar el desempeño laboral de los trabajadores durante el periodo 2020-2021. Quito: Universidad Internacional Sek del Ecuador

Vivaldi, F., y Barra, E. (2012). Bienestar psicológico, apoyo social percibido $\mathrm{y}$ percepción de salud en adultos mayores. Terapia psicológica, 30(2), 23-29

Yactayo, G. (2015. Aplicación de los instrumentos para la evaluación del riesgo psicosocial, en la mejora continua a la prevención y control en una organización. Lima: Universidad Nacional Mayor de San Marcos 